Received Date : 01-May-2016

Revised Date : 12-Jun-2016

Accepted Date : 23-Jun-2016

Article type : Original Article

\title{
Selection process can improve the outcome in locally advanced and recurrent colorectal cancer: activity and results of a dedicated multidisciplinary colorectal cancer centre.
}

Christos Kontovounisios MD, $\mathrm{PhD}^{12}$, Emile Tan MD, FRCS ${ }^{2}$, Nikhil Pawa MD ${ }^{1}$, MRCS, Gina Brown MD, $\mathrm{FRCR}^{3}$, Diana Tait $\mathrm{MD}, \mathrm{FRCP}^{4}$, David Cunningham $\mathrm{MD}, \mathrm{FRCP}^{5}$, Shahnawaz Rasheed PhD, FRCS ${ }^{12}$, Paris Tekkis MD, FRCS ${ }^{12}$.

1. Department of Colorectal Surgery, The Royal Marsden Hospital, Chelsea, London, UK.

2. Department of Surgery and Cancer, Imperial College, London, UK

3. Department of Radiology, The Royal Marsden Hospital, Chelsea, London, UK

4. Department of Clinical Oncology, The Royal Marsden Hospital, Chelsea, London, UK

5. Gastrointestinal and Lymphoma Unit, The Royal Marsden Hospital, Chelsea, London, UK

\section{Corresponding author $\&$ author responsible for reprints:}

Mr Shahnawaz Rasheed PhD FRCS,

Consultant Surgeon

Department of Colorectal Surgery, The Royal Marsden Hospital, Fulham Road, London SW3 $6 \mathrm{JJ}$

Email: s.rasheed@imperial.ac.uk

Tel: 02078082195

This article has been accepted for publication and undergone full peer review but has not been through the copyediting, typesetting, pagination and proofreading process, which may lead to differences between this version and the Version of Record. Please cite this article as doi: 10.1111/codi.13517

This article is protected by copyright. All rights reserved. 


\title{
Acknowledgement
}

This work has been supported by the RMH/ICR Biomedical Research Council and Imperial College.

Article type - Original article

Conflict of interest - none

\begin{abstract}
Aim: There is wide disparity in the care of patients with multi-visceral involvement of rectal cancer. The results of treatment of advanced and recurrent colorectal cancer are presented from a centre where a dedicated Multidisciplinary Team (MDT) is central to the management.
\end{abstract}

Method: All consecutive MDT referrals between 2010 and 2014 were examined. Analysis was undertaken of the referral pathway, site, selection process, management decision, R0 resection rate, mortality / morbidity / Clavien-Dindo (CD) classification of morbidity, length of stay (LOS), and improvement of quality of life.

Results: There were 954 referrals. These included locally advanced primary rectal cancer (LAPRC b-TME) [39.0\%], rectal recurrence (RR) [22.0\%], locally advanced primary colon cancer (LAPCC T3c/d-T4) [21.1\%], colon cancer recurrence (CR) 12.4\%, locally advanced primary anal cancer (LAPAC-failure of CRT/ T3c/d-T4) [3.0\%] and anal cancer recurrence (AR) [2.2\%]. Among these patients 271 operations were performed, 212 primary and 59 for recurrence. These included 16 sacrectomies, 134 total pelvic exenterations) and 121 other multi-visceral exenterative procedures. An R0 resection (no microscopic margin involvement) was achieved in $94.4 \%$ and R1 (microscopic margin involvement) in $5.1 \%$. In LAPRC b-TME the R0 rate was $96.1 \%$ and for RR it was $79 \%$. The length of stay (LOS) varied from 13.3-19.9 days. RR operations had the highest morbidity (Clavien-Dindo [CD] 
1-2 33.3\%) and LAPRC operations had the highest rate of CD 3-4 complications (18.4\%). Most (39.6\%) of the referred patients were from other UK hospitals

Conclusion: Advanced colorectal cancer can be successfully treated in a dedicated referral centre, achieving R0 resection in over $90 \%$ with low morbidity and mortality. Implementation of a standardised referral pathway is encouraged.

\section{What does this paper add to the existing literature?}

The paper demonstrates that advanced primary and recurrent colorectal cancer can be successfully treated by multidisciplinary team management resulting in high rates of complete tumour excision over $90 \%$ and low mortality and morbidity. A structured selection process can improve the outcome through standardised approaches to service delivery to provide the highest quality of care.

\section{Introduction}

Colorectal cancer (CRC) accounts for $13 \%$ of all cancers and is the third most frequent cancer in the 28 countries of the European Union after breast and prostate. It is the second most frequent cause of death after lung cancer [1]. Although the incidence of colon cancer is declining with improved surveillance and prevention of polyp progression, the incidence of rectal cancer in younger patients is increasing by $2.6 \%$ a year in the United States [2]. In the United Kingdom, $33 \%$ of the 14,000 newly diagnosed rectal cancers per year will be locally advanced on presentation $[3,4]$. If left untreated the prognosis is poor with a median survival of less than 1 year and a 5-year survival of less than 5\% [5-7].

Total mesorectal excision (TME) combined with neoadjuvant radiotherapy has led to margin negative rates approaching $90 \%$ and local recurrence rates of between $4 \%$ and $10 \%[3,8,9]$. Although radiotherapy and chemotherapy can downstage cancers so that conventional TME 
will allow complete surgical resection, approximately $6 \%$ of rectal cancers will still be found to have invaded adjacent structures (stage-T4) [3, 9]. There is enough evidence in the literature describing the natural history of locally advanced primary or recurrent colorectal cancer and, however is no established management protocol for these patients regarding the timing of surgery. Despite the important role of chemo/radiotherapy in palliation and symptom control of locally extensive rectal cancer, surgical resection involving a multivisceral or exenterative approach, is the only potentially curative option $[10,11]$. Previous irradiation, distorted mesorectal planes and aggressive tumour biology can, however, lead to a higher risk of $\mathrm{R} 1$ or $\mathrm{R} 2$ resection defined as residual microscopic or macroscopic tumour after surgery, a high rate of postoperative adverse events, varying survival rates and increased difficulty of future salvage surgery [12-14].

Currently there is a wide disparity in care for patients with multivisceral involvement from primary locally advanced and recurrent rectal cancer. The referral process and access to high quality care has been inconsistent and patients may be denied potentially curative surgery or experience delay in being considered for surgery which can result in a poorer outcome. Data collection is variable as patients are not generally included in national databases and outcome reporting is dependent on audits and publications from individual centre.

The aim of the present study was to determine the impact of a multidisciplinary input in the selection process and outcome of pelvic exenteration and to report the experience of a multidisciplinary team dedicated to the treatment of advanced and recurrent colorectal cancer.

This article is protected by copyright. All rights reserved. 


\section{Method}

\section{Patients and the Multidisciplinary Team}

Data of patients with locally advanced rectal cancer referred to the Colorectal Department of the Royal Marsden Hospital between January 2010 and December 2014 were included. They had undergone exenterative multi-visceral surgery for locally advanced primary rectal cancer beyond TME (LAPRC b-TME), rectal recurrence (RR), locally advanced primary colon cancer (LAPCC), colon recurrence (CR), locally advanced primary anal cancer (LAPAC) and anal recurrence (AR). All patients were discussed and assessed by the multidisciplinary team which included medical oncologists, colorectal surgeons, radiotherapists, radiologists, histopathologists, oncology and surgical specialist nurses. Plastic surgeons, urologists, gynaecologists and vascular surgeons were consulted when necessary but were not regular members of the MDT. Meetings were held weekly and the minutes were recorded by an MDT coordinator.

\section{End Points}

The primary end point was the indications and outcome of the total number of patients referred for consideration of exenterative procedures. Those assessed and not operated on were determined and the total number of operations performed was identified. The main secondary endpoints were resection margin status, length of stay (LOS), perioperative adverse events (Clavien-Dindo (15) complications 1-4), 30-day morbidity, 30-day mortality, re-admissions and referral pathway. Histopathological examination of the resected specimen was routinely performed to confirm the diagnosis and determine the anatomical pathology including the status of the resection margin. 


\section{Definitions}

The following definitions were used:

LAPRC b-TME: locally advanced primary rectal cancer. These included patients with locally advanced primary rectal cancer beyond TME. They were identified by MRI which predicted the need for an extended surgical resection beyond the TME plane to achieve an R0 resection.

RR: rectal recurrence. These were cases with recurrence, progression or development of new sites of tumour in the pelvis after previous resectional surgery for rectal cancer.

LAPCC: locally advanced primary colon cancer. This category included patients with locally advanced primary colon cancer, pT3c/d-T4.

CR: colonic recurrence,. These were cases with recurrence, progression or development of new sites of tumour in the abdomen after previous resectional surgery for colon cancer.

LAPAC: locally advanced primary anal cancer. These included patients with anal cancer for whom chemoradiotherapy had failed to ablate the tumour.

AR: anal recurrence. These included patients with recurrence, progression or development of new sites of anal tumour after previous resectional surgery for anal cancer.

\section{Pathological resection margin status:}

- R0: microscopically clear resection margins of at least $1 \mathrm{~mm}$.

- R1: microscopically involved resection margin or tumour within $1 \mathrm{~mm}$ of the resection margin.

- R2: macroscopically involved resection margin.

This article is protected by copyright. All rights reserved. 


\section{Results}

In all, 954 referrals were made between January 2010 and December 2014 from other UK hospitals [39.5\%], hospitals within our network [28.9\%], general practice [14.0\%], general practice within our network [6.9\%] and overseas [10.4\%]In 2010 there were 136, 2011 were 185, 2012 were 213, 2013 were 193 and 2014 were 227 referrals. They were divided as follows: locally advanced primary rectal cancer (LAPRC b-TME) [39.0\%], rectal cancer recurrence (RR) [22.0\%], locally advanced primary colon cancer (LAPCC T3c/d-T4) [21.1 $\%$ ], colon cancer recurrence (CR) [12.4\%], locally advanced primary anal cancer (LAPACfailure of CRT) [3.0\%] and anal cancer recurrence (AR) [2.2\%] (Table 1). Of these 271 patients underwent surgery including 212 for primary cancer (2010-26 operations,2011-46 operations, 2012-44 operations, 2013-50 operations, 2014-46 operations) and 59 for recurrence(2010- 7 operations,2011-12 operations, 2012-14 operations, 2013-6 operations, 2014- 20 operations). Most (56.8\%) were LAPRC. The percentage of patients operated on fell from $35.9 \%$ in 2010 to $28.3 \%$ in 2014 indicating increasing case selection by the MDT. The rate of $\mathrm{R} 1$ resection decreased even though the number of operations performed for LAPRC and RR increased from 23 per year in 2010 to 46 per year in 2014.(Fig.1,2)

There were 16 sacrectomies, 134 total pelvic exenterations and 121 other multi-visceral procedures (Table 2). An R0 resection was achieved in $94.4 \%$ of all cases, with R1 and R2 resections accounting for $5.1 \%$ and $0.3 \%$.In 2010 the R0 resection rate was $93.9 \%, 2011$ was $94.8 \%, 2012$ was $94.8 \%, 2013$ was $89.2 \%$ and 2014 was $98.4 \%$. The rate of R0 resection among LAPRC and RR was $93 \%$ with $\mathrm{R} 1$ and $\mathrm{R} 2$ resection comprising $6.4 \%$ and $0,5 \%$. The $\mathrm{R} 0$ resection rate for $\mathrm{LAPCC}$ and $\mathrm{CC}$ was $98.6 \%$ with $\mathrm{R} 1$ and $\mathrm{R} 2$ being $1.4 \%$ and zero. In the case od LAPAC and AR the R0, R1 and R2 rates were 90\%, 10\% and zero (Table 1). The average length of stay (LOS) was 19.9 days for LAPRC, 19.1 days for RR, 13.3 days for

This article is protected by copyright. All rights reserved. 
LAPCC, 15.1 days for CR, 15.6 days for LAPAC and 14.5 days for AR. Clavien -Dindo 1-2 (CD) complications occurred in 33.3\% of RR procedures and CD 3-4 complications occurred in $18.4 \%$ of LAPRC operations (Table 3). There was no 30 or 90 day mortality.

\section{Discussion}

The goal of surgery for locally advanced rectal cancer is to achieve complete resection of with clear histopathological margins $[16,17]$. Pelvic multi-visceral exenterative procedures were initially described by Brunschwig in 1948 for palliation of advanced cervical cancer [18]. These procedures have evolved to resect pelvic malignant disease radically and when performed as a total pelvic exenteration (TPE), the procedure involves the en bloc resection of the pelvic tumour along with any invaded viscera including the rectum, distal colon, bladder, reproductive organs, lymph nodes and pelvic peritoneum. In some cases, the resection of muscles, ligaments and parts of the pelvic bone may be necessary and the dissection can involve the pelvic side wall muscles, blood vessels and lymph nodes. Such major surgery has resulted in a high rate of postoperative complications and positive resection margins with varying survival rates and long-term functional disability [12-14]. Over time, improved patient selection and advances in imaging, surgical technique, perioperative care and a multidisciplinary team (MDT) cooperation have led to reduced surgical mortality and morbidity [19, 20].

Studies have shown that MDT input in complex diseases results in greater patient satisfaction [21], changes in management [22-24], improved staging [25, 26], increased surgical experience [27] and increased survival [22, 23, 25-30]. It is also well established that surgery by trained specialists especially in rectal cancer results in an improved outcome, [31-34]. The 
identification of patients eligible for surgery is a key element in optimizing the surgical results and survival [35].

In the present series the rate of $\mathrm{R} 0$ resection at $94.4 \%$ was high. This had improved over time. The number of referrals for locally advanced primary rectal cancer and rectal recurrence increased from 64 per year in 2010 to 162 per year in 2014. The percentage of patients operated on fell from $35.9 \%$ in 2010 to $28.3 \%$ in 2014 indicating increasing case selection by the MDT. The rate of R1 resection decreased even though the number of operations performed for LAPRC and RR increased from 23 per year in 2010 to 46 per year in 2014 . This was accompanied by more rigorous selection of patients for surgery despite the increased number of referrals and is ascribable to the effect of the formal multidisciplinary approach.

In contrast some centres treating locally advanced rectal cancer and other pelvic malignancy may apply different patient selection criteria for pelvic exenteration according to the experience and preference of the clinicians [36]. The indications to proceed with pelvic exenterative surgery for advanced rectal cancer include the confinement of the malignant process to the pelvis and the absence of unresectable metastatic disease. Preoperative evaluation with MRI and computed tomography (CT) with positron emission tomography (PET/CT) allow the assessment and characterization of patients with inoperable metastases who will therefore usually not be offered exenterative surgery $[37,38]$.

Contraindications to pelvic exenterative surgery include anatomical, surgical, medical and psychological factors. There is a high degree of variability between treatment centres regarding relative contradictions such as the presence of distant metastases, metastases to para-aortic and/or supra-diaphragmatic lymph nodes, tumour fixation in multiple sites in the pelvis, unpredictable resection margins and tumour involving sacrum at level S1-2 [39, 40]. 
There appears to be general agreement on the absolute contraindications not to proceed with surgery including inoperable metastatic disease, tumour involving bone above S1, unfitness for surgery and the patient's decision not to go ahead. Some centres do not have definite contraindications based on local involvement of the tumour., but MRI demonstrating that an $\mathrm{R} 0$ resection is possible technically, is a reliable guide to the success of exenteration surgery [41].

Until recently there had been no standardisation of definitions with the consequence that accurate comparison of results between different units was not possible. An attempt has been has been made to address this with the publication in 2013 of the "Consensus statement on the multidisciplinary management of patients with recurrent and primary rectal cancer beyond total mesorectal excision (TME) planes" [42]. Three years later it is however, still not clear how the service is currently being provided in the UK. There are still significant delay in the referral of patients and widespread geographical variation in the quality of service delivered. A proposed surgical algorithm for the management of extensive primary and recurrent rectal cancer is suggested in Figures 3 and 4.

It is expected that standardisation of referral criteria, improved access to services, better coordination of care and careful assessment of individual patients through a dedicated complex colorectal cancer MDT should result in significant benefits to patients requiring pelvic exenteration. The treatment of locally advanced and recurrent rectal cancer is costly at all stages of the management pathway and further research is required to assess the costeffectiveness of the service. It is, however, important to recognise that surgical resection is the only modality which can offer a cure and in most cases patients are very keen to have surgery even if very extensive. Streamlined, standardised and well-communicated management should be able to deliver timely, cost-effective and high quality care . A 
structured selection process can improve the outcome of surgery through a standardised approach to service delivery.

Table 1. Total number of referrals, operations and R0, R1 and R2 rates of patients with locally advanced rectal cancer treated between 2010 and 2014 .

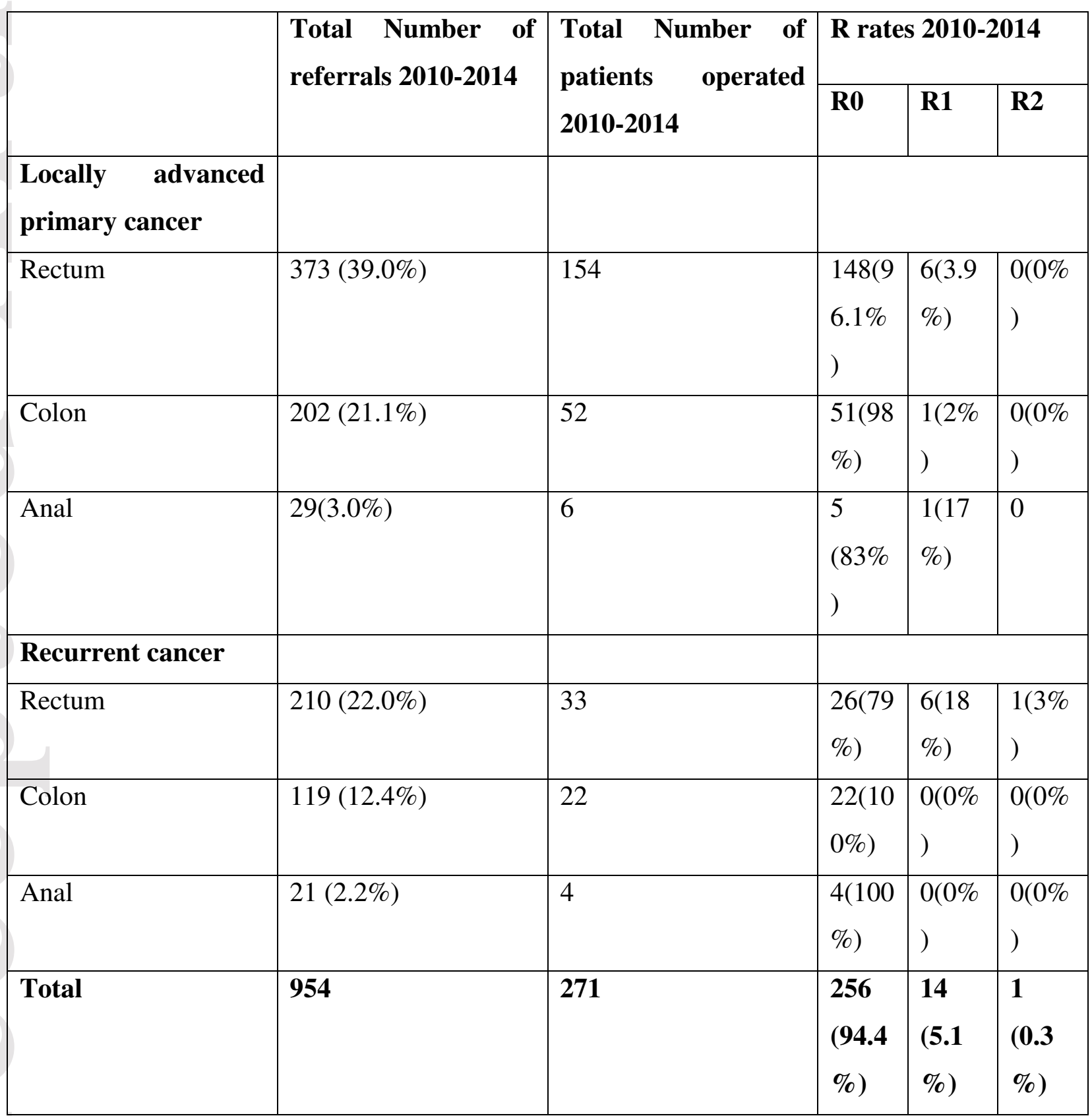

This article is protected by copyright. All rights reserved. 
Table 2. Type of operations for primary and recurrent cancer among 271 patients undergoing surgery between 2010 and 2014

TPE=total pelvic exenteration

\begin{tabular}{|l|l|l|l|}
\hline & SACRECTOMY & TPE & $\begin{array}{l}\text { OTHER } \\
\text { EXENTERATION }\end{array}$ \\
\hline $\begin{array}{l}\text { Locally } \\
\text { advanced } \\
\text { rectal and } \\
\text { recurrent } \\
\text { rectal cancer }\end{array}$ & 16 & 129 & 42 \\
\hline $\begin{array}{l}\text { Locally } \\
\text { advanced } \\
\text { colon and } \\
\text { recurrent } \\
\text { colon cancer }\end{array}$ & 0 & 1 & 73 \\
\hline $\begin{array}{l}\text { Locally } \\
\text { advanced anal } \\
\text { and recurrent } \\
\text { anal cancer }\end{array}$ & 0 & 4 & 6 \\
\hline Total & $\mathbf{1 6}$ & $\mathbf{1 3 4}$ & $\mathbf{1 2 1}$ \\
\hline
\end{tabular}

This article is protected by copyright. All rights reserved. 
Table 3. Surgical Complications among 271 patients undergoing surgery for advanced or recurrent cancer between 2010 and 2014

\begin{tabular}{|l|l|l|}
\hline & \multicolumn{1}{|c|}{$\begin{array}{c}\text { Morbidity } \\
\text { Clavien-Dindo 1-2 }\end{array}$} & \multicolumn{1}{|c|}{$\begin{array}{c}\text { Morbidity } \\
\text { Clavien-Dindo 3-4 }\end{array}$} \\
\hline $\begin{array}{l}\text { Locally advanced } \\
\text { primary cancer }\end{array}$ & & \\
\hline Rectum & $24 / 154(15.5 \%)$ & $14 / 154(9.1 \%)$ \\
\hline Colon & $7 / 52(13.4 \%)$ & $3 / 52(5.7 \%)$ \\
\hline Anal & $1 / 6(16.6 \%)$ & $0 / 6(0 \%)$ \\
\hline Recurrent cancer & & $3 / 33(9.0 \%)$ \\
\hline Rectum & $11 / 33(33.3 \%)$ & $1 / 22(4.5 \%)$ \\
\hline Colon & $5 / 22(22.7 \%)$ & $0 / 4(0 \%)$ \\
\hline Anal & $1 / 4(25 \%)$ & $\mathbf{7 . 7 \%}$ \\
\hline TOTAL & $\mathbf{1 8 \%}$ & \\
\hline
\end{tabular}

Figure 1. The total number patients with LAPRC+RR undergoing surgery between 2010 2014 expressed as a percentage of the total number of referrals

LAPRC=locally advanced primary rectal cancer

$\mathrm{RR}=$ recurrent rectal cancer

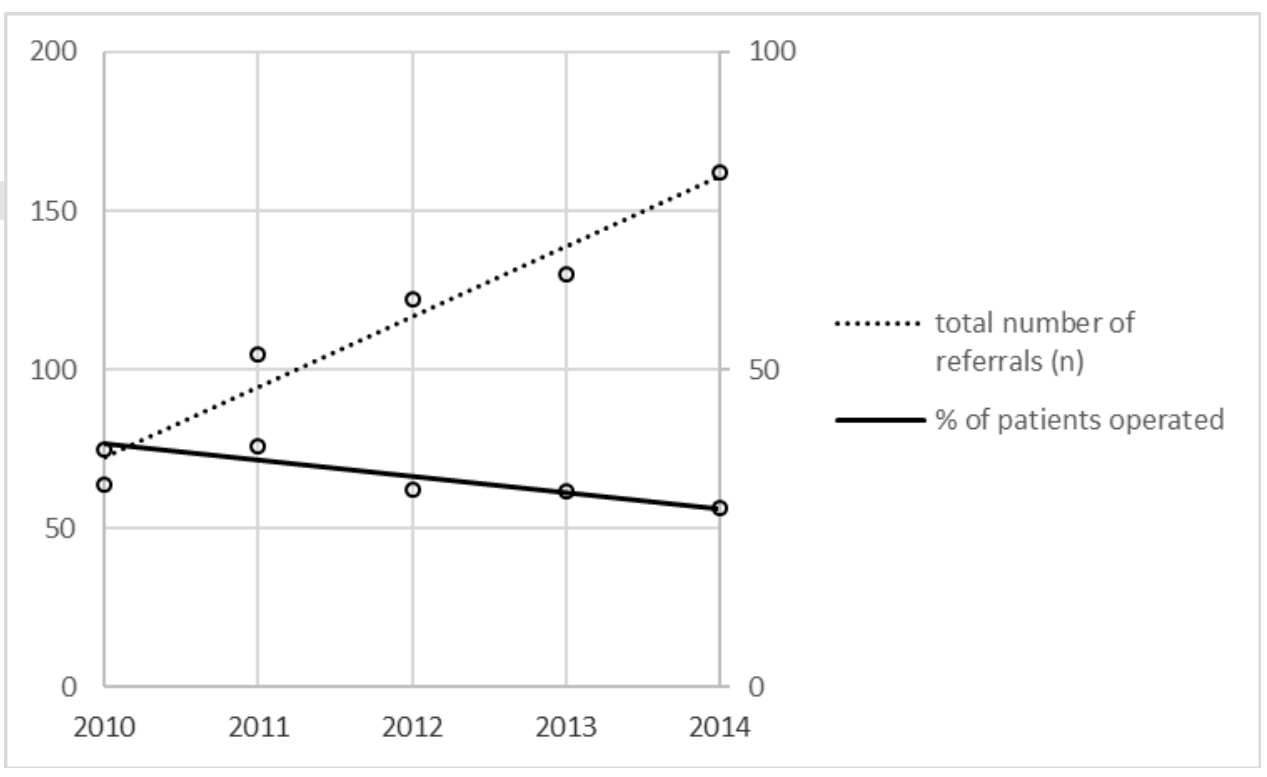

This article is protected by copyright. All rights reserved. 
Figure 2. The total number of operations among 271 patients with LAPRC+RR undergoing surgery between $2010-2014$ including the proportion of R1 resections.

LAPRC=locally advanced primary rectal cancer

$\mathrm{RR}=$ recurrent rectal cancer

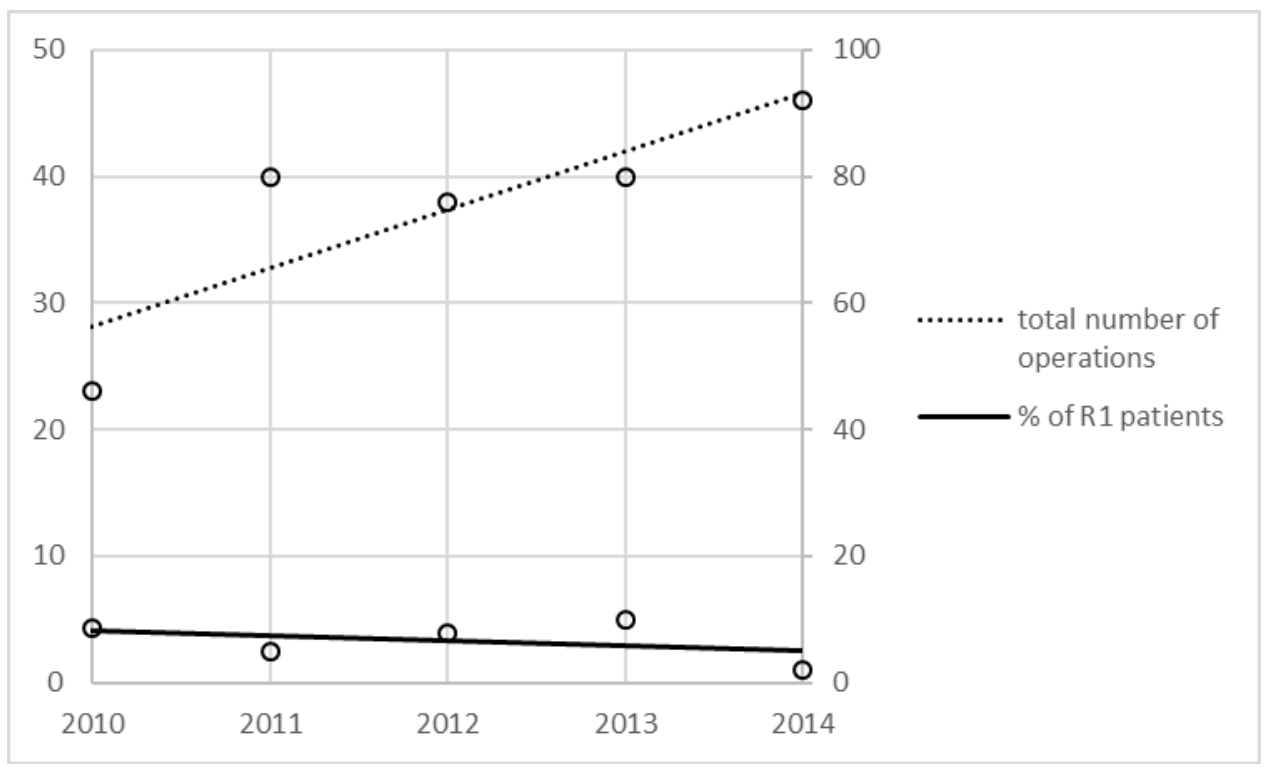

This article is protected by copyright. All rights reserved. 
Figure 3 Locally advanced primary rectal cancer

Algorithm to diagnose and assess resectability of primary rectal cancer beyond total mesorectal excision planes (PRC-bTME).

Boxes in red represent areas of particular controversy:

carcinoembryonic antigen (CEA,), endorectal ultrasound, (EUS) examination under anaesthesia (EUA), magnetic resonance imaging (MRI), computed tomography (CT), thorax, abdomen and pelvis (TAP),, fluorodeoxyglucose (FDG), positron emission tomography (PET), specialist multidisciplinary team (sMDT)
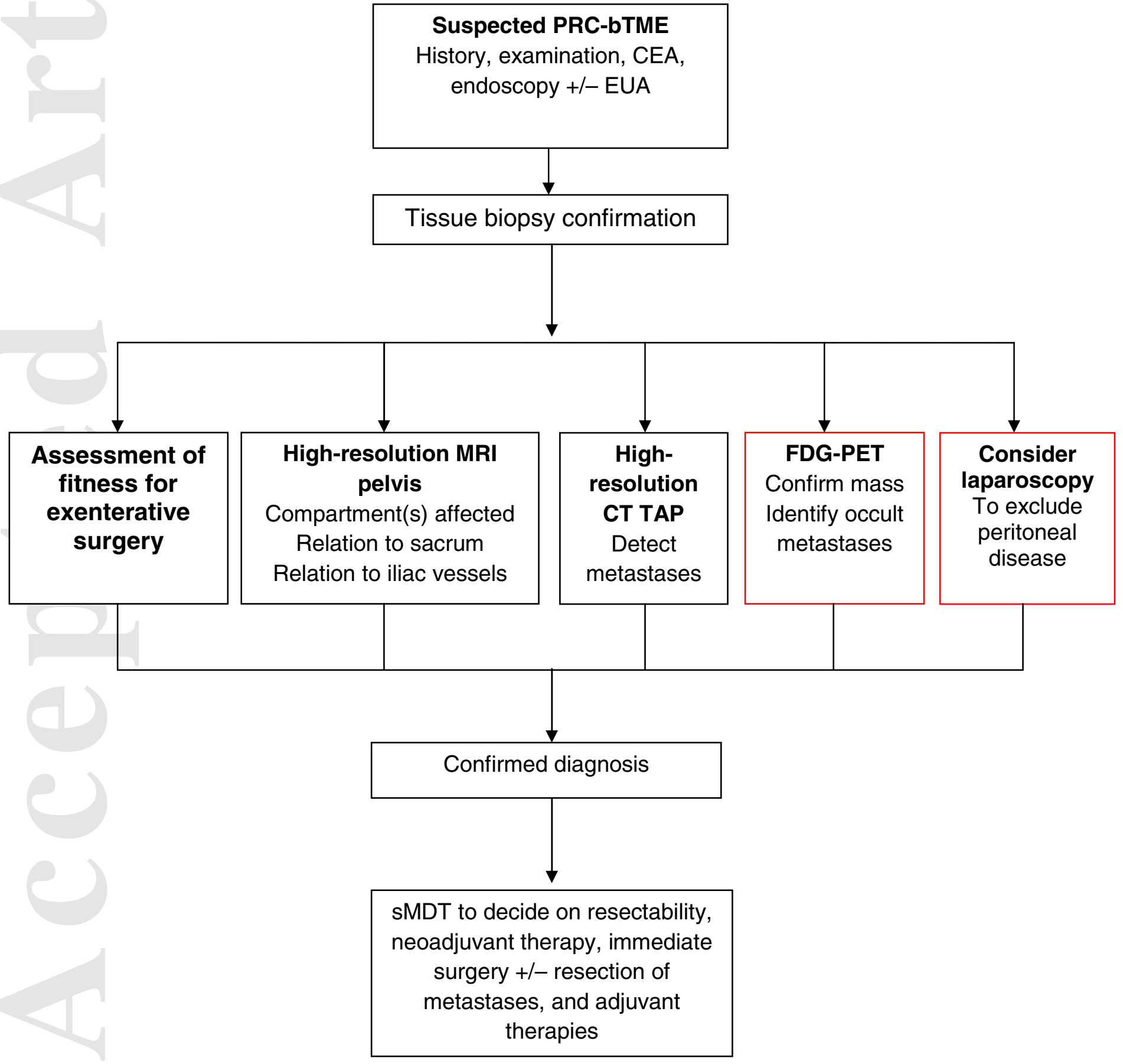

This article is protected by copyright. All rights reserved. 
Figure 4 Recurrent rectal cancer

Algorithm to diagnose and assess resectability of recurrent rectal cancer (RRC).

Boxes in red represent areas of particular controversy:

carcinoembryonic antigen (CEA,), endorectal ultrasound, (EUS) examination under anaesthesia (EUA), magnetic resonance imaging (MRI), computed tomography (CT), thorax, abdomen and pelvis (TAP),, fluorodeoxyglucose (FDG), positron emission tomography (PET), specialist multidisciplinary team (sMDT)

\section{Suspected RRC}

History, examination, CEA, endoscopy +/- EUA

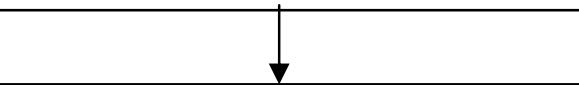

Tissue biopsy confirmation if possible

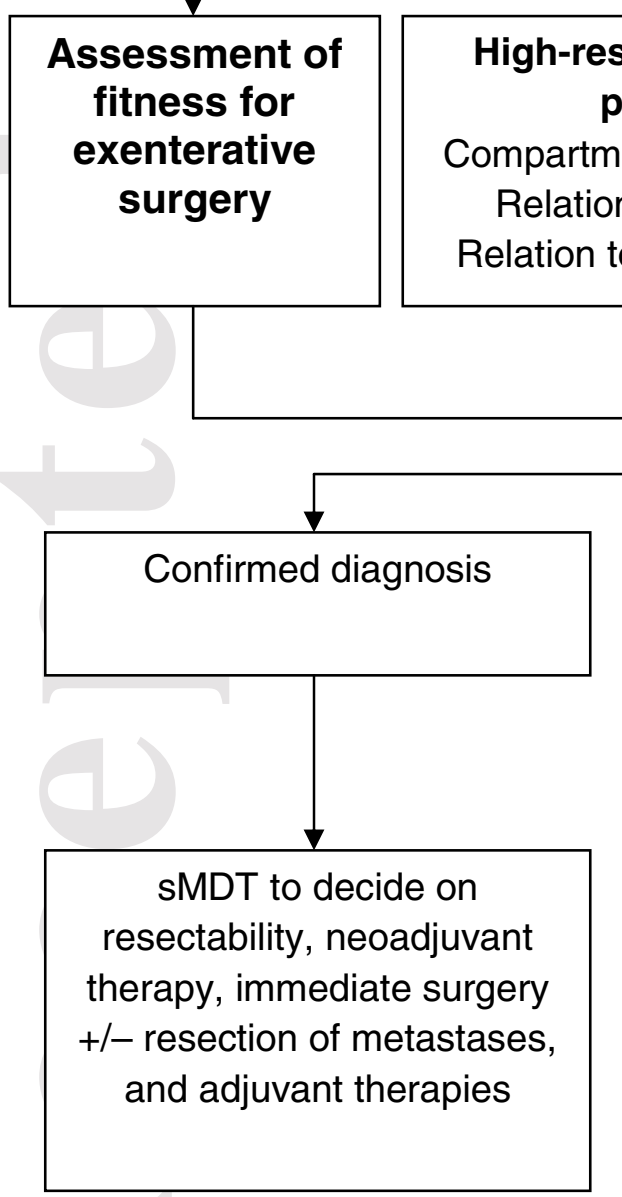

\section{pelvis}

martment(s) affected

Relation to sacrum

Relation to iliac vessels
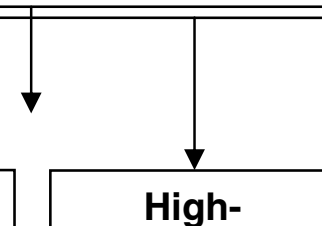
resolution

CT TAP

Detect metastases

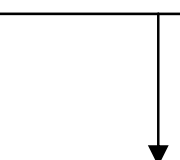

FDG-PET

Confirm mass Identify occult metastases
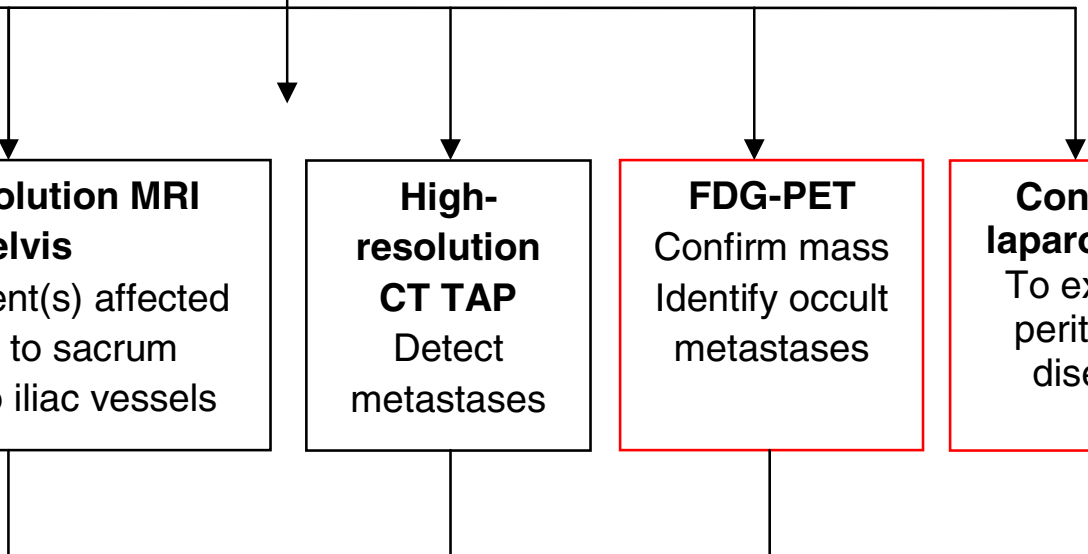

Consider
laparoscopy To exclude peritoneal disease

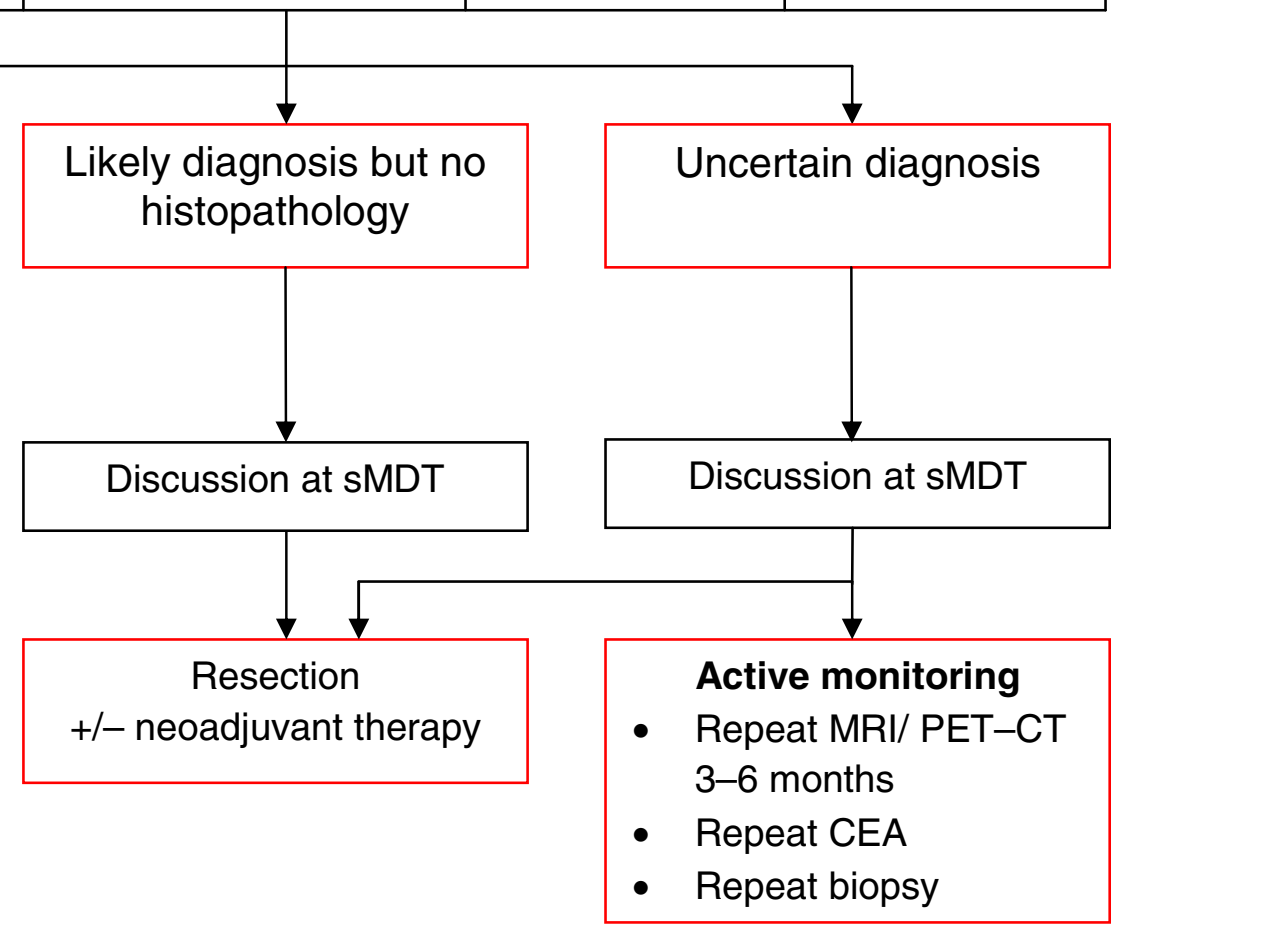

This article is protected by copyright. All rights reserved. 


\section{References}

1. Ferlay, J., et al., Cancer incidence and mortality patterns in Europe: estimates for 40 countries in 2012. Eur J Cancer. 49(6): p. 1374-403.

2. Meyer, J.E., et al., Increasing incidence of rectal cancer in patients aged younger than 40 years: an analysis of the surveillance, epidemiology, and end results database. Cancer. 116(18): p. 4354-9.

3. Group, M.S., Diagnostic accuracy of preoperative magnetic resonance imaging in predicting curative resection of rectal cancer: prospective observational study. BMJ, 2006. 333(7572): p. 779.

4. Cancer services Co-ordinating Group; 2009. .

5. Solum, A.M., R.H. Riffenburgh, and P.A. Johnstone, Survival of patients with untreated rectal cancer. J Surg Oncol, 2004. 87(4): p. 157-61.

6. Carlsson, U., A. Lasson, and G. Ekelund, Recurrence rates after curative surgery for rectal carcinoma, with special reference to their accuracy. Dis Colon Rectum, 1987. 30(6): p. 431-4.

7. Arnott, S.J., The value of combined 5-fluorouracil and $x$-ray therapy in the palliation of locally recurrent and inoperable rectal carcinoma. Clin Radiol, 1975. 26(2): p. 177-82.

8. MacFarlane, J.K., R.D. Ryall, and R.J. Heald, Mesorectal excision for rectal cancer. Lancet, 1993. 341(8843): p. 457-60.

9. van Gijn, W., et al., Preoperative radiotherapy combined with total mesorectal excision for resectable rectal cancer: 12-year follow-up of the multicentre, randomised controlled TME trial. Lancet Oncol. 12(6): p. 575-82.

10. Sagar, P.M. and J.H. Pemberton, Surgical management of locally recurrent rectal cancer. Br J Surg, 1996. 83(3): p. 293-304.

11. Ito, Y., et al., Efficacy of chemoradiotherapy on pain relief in patients with intrapelvic recurrence of rectal cancer. Jpn J Clin Oncol, 2003. 33(4): p. 180-5.

12. Nielsen, M.B., et al., A 10-year experience of total pelvic exenteration for primary advanced and locally recurrent rectal cancer based on a prospective database. Colorectal Dis. 14(9): p. 1076-83.

13. Bhangu, A., et al., Meta-analysis of survival based on resection margin status following surgery for recurrent rectal cancer. Colorectal Dis. 14(12): p. 1457-66.

14. Heriot, A.G., et al., Extended radical resection: the choice for locally recurrent rectal cancer. Dis Colon Rectum, 2008. 51(3): p. 284-91.

15. Dindo, D., N. Demartines, and P.A. Clavien, Classification of surgical complications: a new proposal with evaluation in a cohort of 6336 patients and results of a survey. Ann Surg, 2004. 240(2): p. 205-13.

16. Bhangu, A., et al., Indications and outcome of pelvic exenteration for locally advanced primary and recurrent rectal cancer. Ann Surg. 259(2): p. 315-22.

17. Warrier, S.K., A.G. Heriot, and A.C. Lynch, Surgery for Locally Recurrent Rectal Cancer: Tips, Tricks, and Pitfalls. Clin Colon Rectal Surg. 29(2): p. 114-22.

18. Brunschwig, A., Complete excision of pelvic viscera for advanced carcinoma; a onestage abdominoperineal operation with end colostomy and bilateral ureteral implantation into the colon above the colostomy. Cancer, 1948. 1(2): p. 177-83.

19. Lopez, M.J., S.B. Standiford, and J.L. Skibba, Total pelvic exenteration. A 50-year experience at the Ellis Fischel Cancer Center. Arch Surg, 1994. 129(4): p. 390-5; discussion 395-6.

This article is protected by copyright. All rights reserved. 
20. Courtney, D., et al., Clinical review: surgical management of locally advanced and recurrent colorectal cancer. Langenbecks Arch Surg. 399(1): p. 33-40.

21. Valicenti, R.K., et al., The multidisciplinary clinic approach to prostate cancer counseling and treatment. Semin Urol Oncol, 2000. 18(3): p. 188-91.

22. Morris, E., et al., The impact of the Calman-Hine report on the processes and outcomes of care for Yorkshire's colorectal cancer patients. Br J Cancer, 2006. 95(8): p. $979-85$.

23. Morris, E., et al., The impact of the Calman-Hine report on the processes and outcomes of care for Yorkshire's breast cancer patients. Ann Oncol, 2008. 19(2): p. 284-91.

24. Newman, E.A., et al., Changes in surgical management resulting from case review at a breast cancer multidisciplinary tumor board. Cancer, 2006. 107(10): p. 2346-51.

25. Branagan, G. and N. Davies, Early impact of centralization of oesophageal cancer surgery services. Br J Surg, 2004. 91(12): p. 1630-2.

26. Stephens, M.R., et al., Multidisciplinary team management is associated with improved outcomes after surgery for esophageal cancer. Dis Esophagus, 2006. 19(3): p. 164-71.

27. Granger, S.R., et al., Development of a dedicated hepatopancreaticobiliary program in a university hospital system. J Gastrointest Surg, 2005. 9(7): p. 891-5.

28. Dillman, R.O. and S.D. Chico, Cancer patient survival improvement is correlated with the opening of a community cancer center: comparisons with intramural and extramural benchmarks. J Oncol Pract, 2005. 1(3): p. 84-92.

29. Forrest, L.M., et al., An evaluation of the impact of a multidisciplinary team, in a single centre, on treatment and survival in patients with inoperable non-small-cell lung cancer. Br J Cancer, 2005. 93(9): p. 977-8.

30. Junor, E.J., D.J. Hole, and C.R. Gillis, Management of ovarian cancer: referral to a multidisciplinary team matters. Br J Cancer, 1994. 70(2): p. 363-70.

31. Bokey, E.L., et al., Factors affecting survival after excision of the rectum for cancer: a multivariate analysis. Dis Colon Rectum, 1997. 40(1): p. 3-10.

32. Holm, T., et al., Influence of hospital-and surgeon-related factors on outcome after treatment of rectal cancer with or without preoperative radiotherapy. Br J Surg, 1997. 84(5): p. 657-63.

33. Porter, G.A., et al., Surgeon-related factors and outcome in rectal cancer. Ann Surg, 1998. 227(2): p. 157-67.

34. Read, T.E., et al., Surgeon specialty is associated with outcome in rectal cancer treatment. Dis Colon Rectum, 2002. 45(7): p. 904-14.

35. Troja, A., et al., Surgical options for locally recurrent rectal cancer--review and update. Int J Colorectal Dis. 30(9): p. 1157-63.

36. Bouchard, P. and J. Efron, Management of recurrent rectal cancer. Ann Surg Oncol. 17(5): p. 1343-56.

37. Schurr, P., et al., Radical redo surgery for local rectal cancer recurrence improves overall survival: a single center experience. J Gastrointest Surg, 2008. 12(7): p. 12328.

38. Sagebiel, T.L., et al., Overview of the Role of Imaging in Pelvic Exenteration. Radiographics. 35(4): p. 1286-94.

39. Gannon, C.J., et al., Pelvic exenteration affords safe and durable treatment for locally advanced rectal carcinoma. Ann Surg Oncol, 2007. 14(6): p. 1870-7.

40. Madoff, R.D., Extended resections for advanced rectal cancer. Br J Surg, 2006. 93(11): p. 1311-2.

This article is protected by copyright. All rights reserved. 
41. Moore, H.G., et al., Colorectal cancer pelvic recurrences: determinants of resectability. Dis Colon Rectum, 2004. 47(10): p. 1599-606.

42. Consensus statement on the multidisciplinary management of patients with recurrent and primary rectal cancer beyond total mesorectal excision planes. Br J Surg. 2013 100(8): p. E1-33.

This article is protected by copyright. All rights reserved. 